\title{
Análisis de las dificultades de futuros profesores de química al leer críticamente un artículo de prensa'
}

\author{
Márcia Gorette Lima da Silva" \\ Conxita Marquez Bargallól"' \\ Begonya Oliveras Prat"II
}

I- Agradecimientos al proyecto EDU2015 66643-C2-1-P, a la Coordenação de Aperfeiçoamento Pessoal de Nível Superior (CAPES) y al Programa Ciencias sin Fronteras por el apoyo financiero (Proceso no 4393-139), al grupo LIEC por acoger y contribuir de forma amplia en nuestro estudio y a los futuros maestros de química de la Universidad Federal del Rio Grande del Norte por su participación.

II- Universidade Federal do Rio Grande do Norte,Natal, Brasil.

Contacto: marciaglsilva@yahoo.com.br

III- Universidad Autónoma de Barcelona, Barcelona, España.

Contactos: conxita.marquez@uab.cat:

begona.oliveras@uab.es

IV- El acrónimo desarollado por Bartz (2002) es la síntesis de un conjunto de cuestiones que el lector debe considerar para hacer la lectura crítica. El significado de cada letra es: Claim; Role of the claimant; Information backing the claim; Testing; Independent verification; Conclusion.

\section{Resumen}

En esta investigación se presentan las dificultades que muestran futuros profesores de química de secundaria de una universidad brasileña en analizar críticamente un artículo de prensa con contenido científico. Con esta finalidad se diseñó una actividad de lectura crítica, a partir de una noticia de prensa que hablaba del uso de un producto de limpieza doméstica muy común como producto de belleza. El artículo fue seleccionado con el objetivo que los participantes pudieran aplicar sus conocimientos de química en un contexto real y a la vez desarrollar su capacidad argumentativa. Participaron un total de dieciocho alumnos, nueve del primer año del curso de magisterio y nueve del último. La actividad se realizó en grupos cooperativos fomentando la discusión y la argumentación de las ideas y desde la perspectiva de la participación en prácticas científicas. En concreto, se analizan las dificultades que muestran los futuros profesores a la hora de posicionarse y analizar críticamente la información presente en la noticia a través de sus respuestas a un cuestionariotipo (CRITIC ${ }^{\mathrm{IV}}$ ) y de las transcripciones del debate tras la lectura. Se observa que la capacidad de lectura crítica varía en función del curso. Los alumnos de último curso muestran mayor capacidad de identificar el punto de vista del autor, detectar pruebas en el texto y mostrar acuerdos y desacuerdos razonables después de confrontar la información del texto con sus conocimientos científicos. Sin embargo, muestran dificultades en argumentar de forma fundamentada. No se detectan dificultades en identificar las ideas principales del texto y el propósito del autor en ninguno de los cursos.

\section{Palabras clave}

Argumentación - Lectura crítica - Prensa - Reacción Química - Educación superior. 


\title{
Analysis of the difficulties of preservice teachers of chemistry when critically reading a press article'
}

\author{
Márcia Gorette Lima da Silva" \\ Conxita Marquez Bargallól' \\ Begonya Oliveras Prat"II
}

I- We wish to thank Coordenação de Aperfeiçoamento Pessoal de Nível Superior (CAPES) and Science without Frontiers program for funding (The project EDU-2015 66643-C2-1-P, process n. 4393-13-9), tLIEC group for welcoming and contributing broadly to our study and to preservice teachers of chemistry of Universidade Federal do Rio Grande do Norte for their participation.

II- Universidade Federal do Rio Grande do Norte,Natal, Brasil.

Contacto: marciaglsilva@yahoo.com.br

III- Universidad Autónoma de Barcelona, Barcelona, Spain.

Contact: conxita.marquez@uab.cat;

begona.oliveras@uab.es

IV- The acronym developed by Bartz (2002) is the synthesis of a set of questions that the reader must consider to make critical reading. The meaning of each letter is: Claim; Role of the claimant; Information backing the claim; Testing; Independent verification; Conclusion.

\section{Abstract}

This research presents the difficulties that preservice teachers of chemistry of a Brazilian university show in critically analyzing a newspaper article with scientific content. For this purpose, a critical reading activity was designed, based on a press report about the use of a very common domestic cleaning product as a beauty product (embellishment). The article was selected so that participants could apply their knowledge of chemistry in a real context and at the same time develop their argumentative skills. A total of eighteen students participated, nine of the first year of the teacher training course and nine from the last one. The activity was carried out in cooperative groups promoting discussion of the ideas and argumentation from the perspective of participation in scientific practices. In particular, we analyzed the difficulties that preservice teachers show when expressing their opinion and critically analyzing the information present in the news through their answers to a questionnaire (CRITIC IV) and the transcripts of the debate after the reading. It is observed that critical reading skills vary depending on the course. Last year participants were better able to identify the author's point of view, detect evidence in the text, and show reasonable agreement and disagreement after comparing text information to their scientific knowledge. However, they have difficulties in wellfounded argumentation. No difficulties were detected in identifying the main ideas of the text and the purpose of the author in any of the courses.

\section{Keywords}

Argumentation - Critical reading - Newspaper - Chemical reaction - Higher education. 


\section{Introducción}

Leer críticamente es un proceso fundamental que deberían adquirir todos los alumnos en su escolarización ya que a través de la lectura seguirán aprendiendo a lo largo de toda la vida y el posicionamiento crítico les permitirá analizar la información y actuar. Este trabajo parte de la premisa que conocer las dificultades de leer críticamente textos de contenido científico es el primer paso para ayudar a superarlas.

En este sentido, hay estudios sobre las dificultades de los alumnos de secundaria en leer críticamente (HALKIA; MANTZOURIDIS, 2005; NORRIS; PHILLIPS; KORPAN, 2003; PHILLIPS; NORRIS, 1999) pero hay pocas investigaciones realizadas con futuros profesores (DIAKIDOY et al., 2015).

En el campo de la Didáctica de las Ciencias hay cierto consenso en que uno de los objetivos de la enseñanza y aprendizaje de las ciencias es la construcción y el uso de modelos a través de la participación en prácticas científicas (KELLY, 2008; JIMÉNEZALEIXANDRE; REIGOSA, 2006). Éstas, no se limitan a realizar experimentos, sino que abarcan un conjunto de actividades sociales de construcción del conocimiento y significados, que incluyen la exposición de ideas, su discusión, la evaluación basada en pruebas, como leer, escribir y comunicar tales ideas. En este sentido, las relaciones comunicativas en el aula desempeñan un papel relevante en la enseñanza y aprendizaje (NUSSBAUN, 2002; JIMÉNEZ-ALEIXANDRE; ERDURAN, 2008). El análisis de este sistema de comunicación puede constituir una herramienta importante para identificar los procesos y obstáculos del aprendizaje (JARMAN; MCCLUNE，2010). Es a través de la comunicación que se posibilita la argumentación (KUHN, 2010). Así, la argumentación se entiende como la capacidad de relacionar datos y conclusiones, de evaluar enunciados teóricos en base a datos empíricos o provenientes de otras fuentes. Y es una habilidad imprescindible para poder leer críticamente (OLIVERAS; MÁRQUEZ; SANMARTÍ, 2014).

A pesar de la importancia dada a la argumentación se hacen pocas actividades en el aula de ciencias para promoverla y esto se debe a varios factores. Algunos estudios apuntan a la falta de estrategias pedagógicas (DRIVER et al., 2000; ZEMBAL-SAUL, 2002), de recursos de instrucción (SAMPSON; BLANCHARD, 2012), a un conocimiento pedagógico insuficiente para diseñar las actividades (SIMON et al., 2006) o a las pocas situaciones en que los futuros profesores pueden aprender a argumentar (NEWTON; DRIVER; OSBORNE, 1999).

El Grupo Llenguatge i Ensenyament de les Ciències (LIEC) de la Universidad Autónoma de Barcelona, tiene algunos estudios y propuestas de intervención en aula que contemplan el aprendizaje de las ciencias teniendo como base el desarrollo y transferencia de competencias como leer, interpretar y argumentar a partir de textos de periódicos (OLIVERAS; MÁRQUEZ; SANMARTÍ, 2013; SARDÀ; SANMARTÍ, 2000). Estos estudios muestran que la capacidad lectora de los alumnos viene determinada por el conocimiento de ciencias y por el nivel del desarrollo de su razonamiento (KUHN, 2010). El presente estudio se sitúa en el campo de las investigaciones sobre las prácticas argumentativas en la enseñanza de las ciencias basándose en los estudios para favorecer la competencia científica y lectora a partir de textos periodísticos con contenido científico (OLIVERAS et al., 2014; JARMAN; MACCLUNE, 2010).

El objetivo de esta investigación es analizar las dificultades de los futuros profesores de química en aplicar el pensamiento crítico a la lectura de un artículo de prensa de contenido controvertido y determinar si hay diferencias en función del conocimiento científico.

\section{Argumentación en la clase de ciencias y pensamiento crítico}

Una de las finalidades de la educación de las ciencias es preparar ciudadanos 
críticos capaces de analizar las informaciones utilizando los conocimientos adquiridos en distintas situaciones y eso implica desarrollar el pensamiento crítico (ENNIS, 1996; PAUL; ELDER, 2005; PHILLIPS; NORRIS, 1999; ZOLLER et al., 2000). Esta investigación complementa estudios anteriores sobre las dificultades en leer críticamente textos de periódico con contenido científico (MCCLUNE; ALEXANDER, 2015).

Creemos que es importante que el profesor sea capaz de promover el pensamiento crítico de los estudiantes fomentando la discusión oral y la argumentación en clase de ciencias (OLIVERAS; SANMARTÍ, 2009). Una de las posibilidades es propiciar situaciones en las cuales los alumnos puedan analizar críticamente un texto y evaluar tanto las informaciones, los hechos, los datos como los argumentos presentados.

En las clases de ciencias y en la enseñanza en general, la expresión oral es decisiva, pues el proceso de enseñanza y aprendizaje se desarrolla, en gran parte, por medio del lenguaje (MÁRQUEZ; PRAT, 2005). Para Bakthin (2000) los enunciados, sean de lenguaje oral o escrito, emanan a partir de una actividad humana relacionando el pensamiento cognitivo y el social (VYGOTSKY, 1977). Así, el lenguaje desempeña un papel importante en el aprendizaje, pues es a través de éste que las herramientas culturales y el modo de mirar de una comunidad son puestos a disposición de los individuos (GARRITZ, 2009).

La argumentación es otro aspecto relevante para promover el pensamiento crítico. Los estudios de Kuhn (1993) señalan que la capacidad de emitir argumentos explicativos razonables puede ser considerada como parte del pensar bien, al asumir que la construcción del conocimiento es un proceso permanente de evaluación y los cambios en las conclusiones se deben al surgimiento de nuevos datos y argumentos. Es a través de la interacción con los demás que utilizamos argumentos en diferentes situaciones buscando demostrar o debatir, ofreciendo pruebas, presentando un razonamiento más completo y mejor estructurado, con la finalidad de convencer a alguien de algo. Así pues, analizar el discurso o la comunicación en la clase puede contribuir a profundizar en las preguntas que ayudan a construir conocimiento. Ogborn y otros autores (1998) afirman que es necesario comprender cómo se construyen las explicaciones en el aula, qué dicen los alumnos, explorar los procesos a través de los cuales van siendo construidos los significados en las clases de ciencias y aquellos asociados a las entidades que pueden ser abstractas. Diferentes estudios muestran la potencialidad del discurso argumentativo como espacio de negociación de los significados para la construcción del conocimiento en ciencia y sobre ciencia (ZOHAR, 2007; FISHMAN et al., 2003; STIPCICH et al., 2006; LOUCKS-HORSLEY et al., 2003) en el que el profesor es el agente decisivo (ARCHILA, 2012).

Para que los alumnos sean capaces de explicar un determinado fenómeno necesitan conocimientos científicos para construir y utilizar modelos, además, es necesario tener la capacidad de evaluar criterios que respalden tal explicación (JIMENÉZ-ALEIXANDRE; DÍAZ BUSTAMANTE, 2003) y, consecuentemente, comunicar, leer y escribir textos relacionados con la ciencia (MÁRQUEZ; PRAT, 2005). La capacidad de justificar hechos, argumentar ideas y acciones de forma fundamentada y consciente a partir de modelos teóricos ayuda el desarrollo de la competencia científica. Entendemos el término competencia como la capacidad de movilizar saberes en contextos diversos, analizando los procesos que favorecen la aplicación en un contexto diferente al que fue aprendido (GILBERT; BULTE; PILOT, 2011). De esta forma, la evaluación del conocimiento científico involucra el uso de conceptos científicos necesarios para comprender y ayudar en la toma de decisiones sobre el mundo natural, así como la capacidad de reconocer y explicar cuestiones científicas, someter a comprobación, sacar conclusiones con base científica y comunicarlas, y por lo tanto requiere 
desarrollar el pensamiento crítico del alumnado al dar respuesta a una tarea en particular en un contexto dado (KENNEDY; FISHER; ENNIS, 1991; BAILIN, 2002; NOVAK; DETLOFF, 1989). Otros estudios han demostrado que el trabajo en pequeños grupos ayuda a promover habilidades del pensamiento crítico en la educación superior, a la vez que mejora las habilidades comunicativas (HAGGER et al., 2003).

\section{La formación de profesores en el desarrollo de habilidades en lectura crítica y argumentación}

La literatura actual defiende la importancia de la argumentación en el currículo de ciencias como parte de la alfabetización científica (MCDONALD, 2010). A pesar de ello son pocas las oportunidades propiciadas en aula (SAMPSON; BLANCHARD, 2012) ya que una vez más se demuestra que las reformas políticas no son suficientes para la incorporación de una innovación. Para su incorporación en el aula es necesario un cambio pedagógico del profesorado tanto en el ámbito de la gestión del aula como de los materiales.

Zembal-Saul y otros autores (2002) destacan la importancia de estudios sobre cómo los profesores en ejercicio o aquellos en formación se incorporan a la argumentación científica. Desde esta perspectiva, en los últimos años se han realizado estudios, propuestas y programas de desarrollo profesional (ERDURAN et al., 2006; LEE; LIN, 2005; BRAUND et al., 2013; CETIN et al., 2014; CRIPPEN, 2012; DIAKIDOY et al., 2015; MANGIANTE, 2013; KANG; ANDERSON, 2015; LAWSON, 2010; PLUMMER; OZCELIK, 2015; ABRAMI ET AL., 2015; SAMPSON; GERBINO, 2010; SCHWARZ, 2009; RUIZ ORTEGA; TAMAYO; MÁRQUEZ, 2015). Particularmente Zohar (2007) analiza los medios y condiciones para un cambio pedagógico de los profesores. Su análisis se apoya en tres grupos distintos de estudios: aquellos que sitúan el cambio en la transición de la enseñanza basada en la transmisión a la basada en la construcción del conocimiento; otros que priorizan enseñar habilidades de pensamiento de orden superior y finalmente los que vinculan la enseñanza y la calidad de la argumentación a contextos de conocimiento específico.

Sobre este último grupo, por ejemplo, Cetin y otros autores (2014) analizan la relación de la calidad de los argumentos de futuros profesores y su conocimiento del contenido sobre clonación genética. En su trabajo presentan tres textos que muestran la controversia socio-científica que genera el tema. Los resultados evidencian una relación entre el conocimiento sobre el contenido de clonación y la calidad de los argumentos. Diakidoy y otros autores (2015) analizan el impacto persuasivo de un texto y la evaluación crítica que hacen los futuros profesores. Se observa un cambio significativo del nivel de los argumentos antes y después de la lectura de textos. Además, muestran, entre otros aspectos, que a pesar de evaluar la propuesta como positiva, la mitad confundió la afirmación principal del texto con sus argumentos personales y demostraron poco conocimiento sobre el tema llevando a fragilidades en la evaluación crítica. Estudios como estos señalan la relevancia de planear situaciones durante el periodo de formación de los futuros maestros no solamente para que sepan leer críticamente sino también para prepararlos para el ejercicio de su profesión desde esta perspectiva.

\section{Estrategias para desarrollar la lectura crítica en aula}

Reconocemos la importancia de proporcionar actividades a los alumnos para que desarrollen el pensamiento crítico. Estas actividades requieren el trabajo de habilidades de pensamiento de orden superior, (PHILLIPS; NORRIS, 1999; ZOLLER et al., 2000) lo que implica que los profesores deben estar preparados para diseñar y aplicar actividades que favorezcan el desarrollo de destrezas mentales para la comprensión lectora de sus 
alumnos (OLIVERAS, 2014). Asumimos en este estudio que las habilidades del pensamiento deben ser explícitamente enseñadas en el aula (LINHARES; PASSOS, 2009; ARCHILA, 2012).

Las actividades de lectura crítica de textos periodísticos con contenidos científico que aborden cuestiones complejas se basan en el modelo interactivo en que el lector posee sus ideas o posicionamientos propios y pueden ser distintos del contenido presentado en el texto. Es la interacción entre lector y contenido lo que lleva al procesamiento de la información y a la resignificación de las informaciones.

En términos didácticos, las actividades son desarrolladas en tres fases (SOLÉ, 1998): antes, durante y después de la lectura. Antes de la lectura, se activan las ideas previas y se formulan las hipótesis iniciales; durante la lectura, se regula el proceso de comprensión del texto y análisis crítico del mismo; después de la lectura se evalúa el proceso. Desde esta perspectiva, se revela la importancia de proporcionar en el aula un ambiente de discusión y análisis del problema presentado en el texto, primero individualmente y después socializando y negociando sus ideas en el grupo pequeño $\mathrm{y}$, posteriormente en el gran grupo. Para acompañar y orientar en el desarrollo de la lectura crítica se propone un instrumento orientador (CRITIC) apoyado en los estudios de Bartz (2002) y de Paul y Elder (2005).

\section{Diseño de la investigación}

El estudio fue desarrollado en una universidad pública de Brasil en los estudios de formación de magisterio en Química. La duración de los estudios es de cuatro años con un total de 3.160 horas, contemplando en su currículo asignaturas didáctico-pedagógicas, de contenido específico (química, física, matemática, biología) y otras de formación general. La investigación se llevó a cabo en dos clases de nueve participantes cada. Con un grupo del primer año del curso y con otro de cuarto año. La intervención didáctica se realizó durante 5 días, con un total de diez horas de clase en un programa de extensión del Programa de Formación Complementar del Instituto de Química de esta universidad.

La noticia fue seleccionada porque trataba un contenido de química con informaciones dudosas o que necesitaban un análisis más profundo. El texto involucraba, entre otros, conceptos químicos ya estudiados como cambio químico, propiedades de los materiales, enlace químico, concentración y dilución de disoluciones. También la noticia permitía despertar el interés de los alumnos a partir de exponer la contradicción entre las informaciones presentes en el texto de prensa y en el artículo científico en el que se basaba, el posible impacto social, y la visión ingenua de la construcción del conocimiento implícito en el texto.

El artículo científico en quese basaron para la redacción del texto de prensa fue publicado por el Journal Clinical Investigation (LEUNG et al., 2013) y relataba una investigación impartida por un grupo de científicos de la Universidad de Stanford y del Instituto de Medicina Howard Hughes, USA. Los autores presentaban un estudio preliminar realizado con dos grupos de ratones, unos ratones con dermatitis cutánea aguda provocada por radiación de terapia de cáncer (radioterapia) y unos ratones viejos. Los dos grupos de ratones fueron sometidos al uso tópico (baño) con solución de hipoclorito (en concentración de 0,005\%) y acompañados del factor nuclear (NF-kB) que regula las respuestas celulares de inflamación y envejecimiento. Los autores afirmaban que en estudios anteriores habían verificado que el hipoclorito inhibía la actividad del gen que activa el factor nuclear por medio de la oxidación. Observaron, a nivel microscópico, que en los ratones con dermatitis el uso tópico del hipoclorito reducía la expresión de los genes y la severidad de la enfermedad e impedía la ulceración de la piel. Y en los ratones viejos atenuaba la expresión del gen de reparación de la piel, con incremento de la espesura de la epidermis y su proliferación cuando se comparaba con la piel de animales 
más jóvenes. Los autores reconocían que se trataba de un estudio preliminar y sugerían el uso de hipoclorito con fines clínicos.

Diferentes noticias de prensa divulgadas en blogs de países como Portugal, España, EUA, Inglaterra y Brasil hacían eco de las informaciones presentes en el artículo científico. Algunos de los blogs eran más cuidadosos con la información, pero otros presentaban imágenes que podrían llevar al lector a hacer o reproducir el experimento con el hipoclorito sódico. Éste es una sal derivada del ácido hipocloroso y su utilización más habitual es como hipoclorito de sodio, empleado desde 1792 como desinfectante tópico en función de su propiedad antimicrobiana, la cual depende de la concentración (BORIN; BECKER; OLIVEIRA, 2007). Por ejemplo, soluciones diluidas $(0,0005 \% \mathrm{v} / \mathrm{v})$ son utilizadas en humanos como tratamiento tópico para dermatitis cutáneas.

La noticia Lavarse con lejía puede rejuvenecer, apunta un estudio (AGENCIA HÉLICE, 2013), induce al lector a creer que el uso tópico del hipoclorito de sodio presente en un producto comercial de limpieza traería beneficios de rejuvenecimiento de la piel humana. Para interpretar correctamente el texto es necesario analizar la sustancia en función de sus propiedades químicas y su actuación en términos de la concentración de las disoluciones a partir del producto de uso doméstico comercializado. Además, para verificar la confiabilidad de los datos, es relevante discutir el propio proceso de generación de conocimiento científico, ya que el texto no informaba con claridad que se trataba de un estudio preliminar no desarrollado con humanos.

\section{Descripción de la secuencia de actividades}

Se diseñó una secuencia de actividades estructuradas según las tres fases del proceso lector (antes, durante y después de la lectura). La fase previa consistía en interpretar el título y las imágenes de la noticia y anticipar el posible contenido de la misma. Durante la lectura, a través de cuestionario CRITIC (cuadro 4) se analizaron diferentes aspectos de la noticia, como el contenido científico y el método de investigación presentado. En la fase posterior a la lectura se comparó el texto periodístico con el artículo original a partir del cual se había escrito la noticia y se interpretaron sus posibles implicaciones sociales (Cuadro 1).

Cuadro 1: Actividades realizadas en las tres fases del proceso lector

\begin{tabular}{|c|c|}
\hline Actividad & Descripción de la actividad \\
\hline $\begin{array}{c}\text { Antes de la } \\
\text { lectura }\end{array}$ & $\begin{array}{c}\text { Análisis del título y de las imágenes del texto de } \\
\text { prensa Lavarse con lejía puede rejuvenecer, apunta } \\
\text { un estudio. Discusión de las ideas. }\end{array}$ \\
\hline $\begin{array}{l}\text { Durante la } \\
\text { lectura }\end{array}$ & $\begin{array}{l}\text { Lectura individual y en grupo utilizando el } \\
\text { cuestionario CRITIC para identificar los elementos de } \\
\text { la lectura crítica del texto de prensa. } \\
\text { Socialización de las respuestas. }\end{array}$ \\
\hline $\begin{array}{l}\text { Después de } \\
\text { la lectura }\end{array}$ & $\begin{array}{c}\text { Debate sobre las implicaciones para la sociedad, } \\
\text { de la información presentada después del análisis a } \\
\text { partir del artículo científico original. }\end{array}$ \\
\hline
\end{tabular}

Fuente: Elaboración propia.

Para analizar el pensamiento crítico del alumnado se utilizó el cuestionario elaborado con base al instrumento-guía de lectura CRITIC (BARTZ, 2002) y de los elementos de lectura crítica por Paul y Elder (2005). Éste consiste en trabajar una serie de elementos para ayudar al lector a identificar las ideas principales del texto, a pensar en el interés del autor en escribirlo y a analizar la fiabilidad y validez de las pruebas y argumentos aportados.

Cuadro 2- Elementos de lectura crítica de textos con contenido científico (OLIVERAS MÁRQUEZ; SANMARTÍ, 2013)

\begin{tabular}{|l|}
\hline Elementos de lectura crítica de textos con contenido científico \\
\hline 1. Identificar las ideas principales del texto \\
\hline 2. Identificar el propósito del autor \\
\hline 3. Identificar las suposiciones y el punto de vista del autor \\
\hline 4. Formular una pregunta científica a la que da respuesta el autor \\
en el artículo y/o \\
Diseñar un experimento científico para comprobar la información \\
del texto \\
\hline 5. Identificar datos y evidencias del texto \\
\hline 6. Sacar conclusiones infiriendo a partir de pruebas \\
\hline
\end{tabular}

Fuente: Oliveras; Márquez, Sanmartí (2013). 
Las actividades desarrolladas por los participantes a lo largo de cinco encuentros de dos horas, son presentadas en el cuadro 3. Asimismo, se incluyen las orientaciones del profesor y el tiempo de realización.
La recogida de datos fue realizada combinando las respuestas del cuestionario CRITIC (durante la lectura) y la grabación del debate (después de la lectura). Para facilitar la transcripción de las grabaciones se

Cuadro 3- Descripción de las actividades en cada sesión de clase

\begin{tabular}{|c|c|c|c|c|c|}
\hline & 0 (min) & \multicolumn{2}{|c|}{$30-60$ (min) } & & -120 (min) \\
\hline \multirow[t]{2}{*}{1} & Orientaciones generales & \multicolumn{2}{|c|}{ Actividad individual (autorización del uso en la investigación) } & $\begin{array}{r}\text { Presentación } \\
\text { fundamentan } \\
\text { con la lectura } \\
\text { desarrollar la } \\
\text { objetivo didác } \\
\text { du }\end{array}$ & $\begin{array}{l}\text { los aspectos teóricos que } \\
\text { actividades relacionadas } \\
\text { ítica, la importancia para } \\
\text { mpetencia científica y el } \\
\text { de las tres fases (antes, } \\
\text { te y después). }\end{array}$ \\
\hline & 0 (min) & $15-30$ (min) & $30-60$ (min) & $60-90$ (min) & $90-120$ (min) \\
\hline \multirow[t]{2}{*}{2} & $\begin{array}{l}\text { Orientaciones antes de } \\
\quad \text { la lectura }\end{array}$ & $\begin{array}{l}\text { Actividad individual } \\
\text { (interpretar el título y las } \\
\text { imágenes del texto de } \\
\text { prensa) }\end{array}$ & $\begin{array}{l}\text { Actividad en grupo (discutir las } \\
\text { ideas sobre el título) }\end{array}$ & $\begin{array}{l}\text { Socialización } \\
\text { de las ideas } \\
\text { en el gran } \\
\text { grupo }\end{array}$ & $\begin{array}{l}\text { Debatir y negociar las } \\
\text { ideas } \\
\text { Llegar a un consenso } \\
\text { sobre la idea más } \\
\text { plausible } \\
\end{array}$ \\
\hline & 0 (min) & $15-30$ (min) & $30-60$ (min) & \multicolumn{2}{|c|}{$60-120$ (min) } \\
\hline \multirow[t]{2}{*}{3} & $\begin{array}{l}\text { Orientaciones durante } \\
\quad \text { la lectura }\end{array}$ & $\begin{array}{l}\text { Actividad individual lectura } \\
\text { del texto de prensa }\end{array}$ & Análisis del texto con CRITIC & \multicolumn{2}{|c|}{$\begin{array}{l}\text { Actividad en grupo, discutir las ideas a } \\
\text { partir del CRITIC }\end{array}$} \\
\hline & 0 (min) & $15-45$ (min) & 45-100 (min) & \multirow{2}{*}{\multicolumn{2}{|c|}{$\begin{array}{c}\text { Orientaciones sobre la actividad individual } \\
\text { en el laboratorio de informática de } \\
\text { busca de datos (comparar con el artículo } \\
\text { científico original) }\end{array}$}} \\
\hline \multirow[t]{2}{*}{4} & $\begin{array}{l}\text { Orientaciones de } \\
\text { regulación de la lectura }\end{array}$ & $\begin{array}{l}\text { Recuerdo de los aspectos } \\
\text { principales apuntados en } \\
\text { el texto de prensa, plantear } \\
\text { hipótesis para verificar las } \\
\text { informaciones. }\end{array}$ & $\begin{array}{l}\text { Sistematización sobre las ideas } \\
\text { principales del texto de prensa y } \\
\text { las informaciones necesarias para } \\
\text { buscar y comparar con el artículo } \\
\text { científico original. }\end{array}$ & & \\
\hline & 0 (min) & \multicolumn{2}{|c|}{$15-90$ (min) } & \multicolumn{2}{|c|}{$90-120$ (min) } \\
\hline 5 & $\begin{array}{l}\text { Orientaciones después } \\
\text { de la lectura }\end{array}$ & \multicolumn{2}{|c|}{$\begin{array}{l}\text { Actividad en grupo, discutir las hipótesis levantadas sobre el } \\
\text { interés y aspectos científicos y metodológicos del texto de prensa }\end{array}$} & \multicolumn{2}{|c|}{$\begin{array}{l}\text { Discusión de los datos y conclusiones en } \\
\text { el gran grupo después de la comparación } \\
\text { con del artículo original } \\
\text { Llegar a un consenso sobre las } \\
\text { implicaciones de los datos }\end{array}$} \\
\hline
\end{tabular}

Fuente: Elaboración propia.

utilizó como suporte el software Transana ${ }^{\circledR}$ (WOODS, 2002).

El cuestionario con el acrónimo CRITIC busca orientar al lector en la identificación de las principales afirmaciones del texto, a valorar la solidez de las afirmaciones que presenta, la confiabilidad y validez de las pruebas $\mathrm{y}$ argumentos utilizados. También orienta a detectar incoherencias, imprecisiones, errores o contradicciones (Cuadro 4). Las preguntas en este cuadro fueron orientadoras de las actividades realizadas. Las respuestas a los diferentes apartados del CRITIC y las transcripciones forman parte de los datos que se analizan. 
Cuadro 4: Cuestionario CRITIC

\begin{tabular}{|c|c|c|c|}
\hline & ¿En qué pensar al leer? & Ejemplos de preguntas & Habilidades cognitivas \\
\hline C & $\begin{array}{l}\text { Contenido o declaración } \\
\text { presentada en el texto. }\end{array}$ & $\begin{array}{c}\text { ¿A quién va dirigido el texto? } \\
\text { ¿Qué problema se expone en el texto? } \\
\text { ¿Cuál es la idea principal? } \\
\text { ¿A quién puede interesar su lectura? } \\
\text { ¿Con qué contenidos científicos puede estar } \\
\text { relacionada? }\end{array}$ & $\begin{array}{l}\text { Comprender la idea principal, } \\
\text { seleccionar la información básica y } \\
\text { construir una oración nueva. } \\
\text { Reconocer situaciones de la vida } \\
\text { dotadas de contenido científico. } \\
\text { Pensar en términos de modelos } \\
\text { científicos }\end{array}$ \\
\hline $\mathrm{R}$ & $\begin{array}{l}\text { Rol de la declaración } 0 \\
\text { papel de la declaración. }\end{array}$ & $\begin{array}{l}\text { ¿Cuál el tipo de texto (anuncio, noticia...)? } \\
\text { ¿Por qué el autor lo escribe? } \\
\text { ¿Está usted de acuerdo con lo que está escrito? } \\
\text { ¿El autor sabe del tema? ¿Quién ha escrito este } \\
\text { documento? }\end{array}$ & Identificar el propósito del autor \\
\hline । & $\begin{array}{l}\text { Ideas presentes en el } \\
\text { texto }\end{array}$ & $\begin{array}{c}\text { ¿Qué ideas o creencias pueden estar por detrás de } \\
\text { las declaraciones expresas en el texto? ¿Qué ideas o } \\
\text { creencias llevan al autor a escribir el texto? } \\
\text { ¿Qué ideas expone? }\end{array}$ & $\begin{array}{l}\text { Reconocer que la ciencia no está libre } \\
\text { de ideología. } \\
\text { Identificar el punto de vista del autor }\end{array}$ \\
\hline $\mathrm{T}$ & $\begin{array}{c}\text { Testes de comprobación } \\
\text { o refutación de las } \\
\text { declaraciones }\end{array}$ & $\begin{array}{l}\text { ¿Puede usted proponer un test o experimento para } \\
\text { comprobar lo que fue expuesto en el texto? ¿Cuál? } \\
\text { ¿Cómo sería? } \\
\text { ¿Los datos presentados en el texto son suficientes y } \\
\text { válidos? ¿Por qué? }\end{array}$ & $\begin{array}{l}\text { Aplicar conocimientos científicos para } \\
\text { plantear propuestas alternativas } \\
\text { Formular una pregunta investigable } \\
\text { científicamente. } \\
\text { Identificar y valorar el tipo de prueba que } \\
\text { aporta el autor }\end{array}$ \\
\hline I & Información & $\begin{array}{l}\text { ¿Qué pruebas o evidencias se presentan para apoyar } \\
\text { la declaración del texto? } \\
\text { ¿Las informaciones o datos tienen incoherencias, } \\
\text { errores o contradicciones? }\end{array}$ & $\begin{array}{l}\text { Analizar la información aportada. } \\
\text { Valorar la información a partir de los } \\
\text { conocimientos propios. } \\
\text { Argumentar a favor o en contra de las } \\
\text { pruebas o experimentos aportados. } \\
\text { Juzgar la credibilidad de la fuente }\end{array}$ \\
\hline C & Conclusiones & $\begin{array}{c}\text { ¿Fue usted convencido sobre la declaración del texto? } \\
\text { ¿El texto fornece argumentos suficientes? } \\
\text { ¿El texto está de acuerdo con el conocimiento } \\
\text { científico actual? } \\
\text { ¿Ha aprendido alguna cosa? }\end{array}$ & $\begin{array}{c}\text { Confrontar las conclusiones del texto con } \\
\text { los conocimientos científicos del lector. } \\
\text { Extraer conclusiones basadas en } \\
\text { pruebas. } \\
\text { Argumentar acuerdos y desacuerdos. } \\
\text { Comunicar conclusiones válidas. } \\
\text { Demostrar la conexión y comprensión } \\
\text { de hechos del mundo con fenómenos } \\
\text { científicos } \\
\text { Reflexionar sobre las implicaciones } \\
\text { sociales de la ciencia }\end{array}$ \\
\hline
\end{tabular}

Fuente: Elaboración propia.

* Adaptado de Bartz (2002) y por el grupo LIEC.

\section{Análisis de datos}

Para analizar los datos se han definido una rúbrica con seis categorías, que corresponden a los elementos de lectura crítica de textos con contenido científico y se determinaron sus porcentajes. En cada categoría se utilizaron cinco niveles de argumentos (cuadro 5). 
Cuadro 5- Rúbrica para análisis de los argumentos (OLIVERAS; MÁRQUEZ; SANMARTÍ, 2013)

\begin{tabular}{|c|c|}
\hline Categorías & Nivel \\
\hline $\begin{array}{l}\text { 1: Identificación de las ideas } \\
\text { principales del texto }\end{array}$ & $\begin{array}{l}\text { 1. Citan información no relevante o no reelaboran la información. } \\
\text { 2. Sólo identifican una de las ideas o conceptos clave. } \\
\text { 3. Hacen referencia a más de una idea o concepto clave. } \\
\text { 4. Expresan con sus propias palabras la información más importante. Identifican algunas de las ideas y } \\
\text { conceptos clave que se usan de una manera comprensible. Hacen interacciones entre ideas. } \\
\text { 5. Expresan con sus propias palabras la información más importante de forma comprensible. Identifican } \\
\text { todas las ideas y conceptos clave que se usan de una manera comprensible. }\end{array}$ \\
\hline $\begin{array}{l}\text { 2: Identificación del } \\
\text { propósito del autor }\end{array}$ & $\begin{array}{l}\text { 1. Se cita información irrelevante; } \\
\text { 2. La información expresada no se puede inferir del texto; } \\
\text { 3. Se supone que las noticias solo son para informar de una manera neutra e imparcial; } \\
\text { 4. Se identifica el propósito del autor, pero de manera poco precisa ya sea porque no se redacta bien o } \\
\text { porque no se concreta lo suficiente. } \\
\text { 5. Se comunica bien el propósito que se cree que el autor tiene. } \\
\text { 6. Se nota que el autor, además de informar, tiene otras intenciones. }\end{array}$ \\
\hline $\begin{array}{c}\text { 3: Identificación de las } \\
\text { suposiciones y puntos de vista } \\
\text { del autor }\end{array}$ & $\begin{array}{l}\text { 1. No se contesta o cita información irrelevante, o no se identifica el punto de vista del autor/a; } \\
\text { 2. Se hacen suposiciones no razonables en función de pruebas y no se identifica el punto de vista del autor } \\
\text { ni se justifica el punto de vista del texto } \\
\text { 3. Se citan frases textuales del texto sin inferir el punto de vista del autor/a; } \\
\text { 4. Se hacen suposiciones razonables y se identifica el punto de vista del autor/a, pero no es justificado; } \\
\text { 5. Se hacen suposiciones razonables, se identifica y justifica el punto de vista del autor/a a partir del texto. }\end{array}$ \\
\hline $\begin{array}{l}\text { 4: Formulación de una pregunta } \\
\text { científica o diseño de un } \\
\text { experimento para comprobar la } \\
\text { información del texto }\end{array}$ & $\begin{array}{l}\text { 1. Se plantean preguntas poco coherentes o experimentos irrelevantes; } \\
\text { 2. Se plantean la pregunta sin concreciones o realizan experimentos demasiado generales. } \\
\text { 3. Se preguntan cuestiones a las que no responde el texto o realizan experimentos dirigidos únicamente a } \\
\text { entender el porqué del problema. } \\
\text { 4. Se formulan preguntas fundamentadas e importantes desde la ciencia analizando solo una de las } \\
\text { variables, o se realiza un experimento para comprobarlo basado en una de las variables; } \\
\text { 5. Se formulan preguntas fundamentadas e importantes desde la ciencia analizando todas las variables a } \\
\text { considerar, o se realiza un experimento para comprobarlo basado en todas las variables. }\end{array}$ \\
\hline $\begin{array}{l}\text { 5: Identificación de datos y } \\
\text { pruebas }\end{array}$ & $\begin{array}{l}\text { 1. Se valida la información por confianza (no se juzga la credibilidad de la fuente) porque se cree que el } \\
\text { autor/a ha presentado las informaciones en el texto. } \\
\text { 2. Se citan informaciones del texto con un razonamiento no elaborado e impreciso o bien se sacan } \\
\text { conclusiones basadas en informaciones del texto no relevantes y no se refieren si son evidencias o no. } \\
\text { 3. Se hace referencia a si el texto da pruebas o no, o si las informaciones que aporta tienen validez } \\
\text { científica sin más explicaciones, o bien dan argumentos poco elaborados o sin buscar ninguno para validar } \\
\text { la información del texto. } \\
\text { 4. Se sacan conclusiones fundamentadas a partir de la información aportada por el texto (hecho, datos, } \\
\text { pruebas, etc.), sin distinguir el tipo de fuente (hecho, opinión, argumento científico, etc.). } \\
\text { 5. Se hace distinción entre hechos, argumentos científicos y opiniones del texto. Se saca conclusiones } \\
\text { teniendo en cuenta la información de que se dispone y por un razonamiento sensato demuestran } \\
\text { capacidad para analizar y evaluar la información objetivamente. }\end{array}$ \\
\hline $\begin{array}{l}\text { 6: Argumentación de } \\
\text { conclusiones a partir } \\
\text { de pruebas }\end{array}$ & $\begin{array}{l}\text { 1. Se citan argumentos irrelevantes; } \\
\text { 2. Se llega a conclusiones a partir de conocimientos cotidianos sin activar los conocimientos científicos. } \\
\text { 3. Utilizan sus conocimientos de la ciencia y muestran capacidad para argumentar acuerdo o desacuerdo y } \\
\text { no confrontan sus conocimientos con la información del texto. } \\
\text { 4. Confrontan la información del texto con los conocimientos científicos y muestran sus acuerdos o } \\
\text { desacuerdos razonables sin fundamentarlos explícitamente. } \\
\text { 5. Confronta la información del texto con los conocimientos científicos y muestran capacidad de } \\
\text { argumentar de forma fundamentada. }\end{array}$ \\
\hline
\end{tabular}

Fuente: Oliveras, Márquez, Sanmartí (2013). 
Las respuestas y las transcripciones se organizaron en tablas y se analizaron en función de las categorías expresas en el cuadro 5 en un proceso inductivo-deductivo. Cada unidad de significado fue asignada a una categoría y a un nivel (elaboradas a priori) y se identificaron sus porcentajes (BARDÍN, 1986; OLIVERAS; MÁRQUEZ; SANMARTÍ, 2014).

\section{Resultados}

El análisis de los datos muestra un primer diagnóstico sobre algunas dificultades de los futuros profesores de química al realizar la lectura crítica y movilizar sus conocimientos. Durante el análisis del título fue posible observar sus inferencias sobre los aspectos generales del texto como, por ejemplo, la autoría, a quién está dirigido y el tipo de texto. El siguiente extracto ${ }^{1}$ ilustra tales características:

F4 ( $1^{\circ}$ año): El título probablemente se refiere a un artículo científico sobre los beneficios que la lejía puede traer para rejuvenecer. Aparentemente está dirigido a personas interesadas en el embellecimiento.

Otros presentan aspectos sobre el método para alcanzar el objetivo del tema, los posibles contenidos científicos involucrados e implicaciones para el lector, como por ejemplo:

F6 ( $4^{\circ}$ año): A partir del título creo que el texto abordará las influencias de la sustancia en la piel de los seres humanos. El autor ha preferido utilizar la palabra lejía pues su público son lectores que no están familiarizados con la nomenclatura química. Creo que su opción por este término fue para causar impacto, pues esta sustancia tiene la propiedad de basicidad elevada y puede provocar quemaduras en la piel.

A partir de las respuestas de los cuestionarios (CRITIC) y de las intervenciones de los alumnos en el audio se obtuvo la frecuencia en cada categoría para los dos grupos.

Tabla 1- Niveles de argumentos de los participantes

\begin{tabular}{|c|c|c|c|c|c|c|c|c|c|c|c|c|}
\hline & \multicolumn{2}{|c|}{ Categoría 1} & \multicolumn{2}{|c|}{ Categoría 2} & \multicolumn{2}{|c|}{ Categoría 3} & \multicolumn{2}{|c|}{ Categoría 4} & \multicolumn{2}{|c|}{ Categoría 5} & \multicolumn{2}{|c|}{ Categoría 6} \\
\hline & $1^{\circ}$ año & $4^{\circ}$ año & $1^{0}$ aก̃o & $4^{0}$ año & $1^{\circ}$ año & $4^{\circ}$ año & $1^{0}$ año & $4^{\circ}$ aก̃o & $1^{0}$ aก̃o & $4^{0}$ año & $1^{0}$ año & $4^{\circ}$ año \\
\hline Niv.1 & & & & & 12,5 & & 25,0 & 12,5 & & & & \\
\hline Niv.2 & 25,0 & & & & & & 75,0 & 62,5 & 100,0 & 50,0 & 62,5 & \\
\hline Niv.3 & 75,0 & 25,0 & 87,5 & 25,0 & 25,0 & 37,5 & & 25,0 & & 25,0 & 37,5 & 25,0 \\
\hline Niv.4 & & 75,0 & 12,5 & 75,0 & 62,5 & 25,0 & & & & & & 75,0 \\
\hline Niv.5 & & & & & & 37,5 & & & & 25,0 & & \\
\hline
\end{tabular}

Fuente: Elaboración propia.

A continuación, detallamos cada categoría.

\section{Categoría 1: Identificación de las ideas principales del texto}

En esta categoría la mayoría de los participantes del $1^{\circ}$ año hacen referencia a más

1- Todos los extractos presentes en este artículo son de entrevistas que pertenecen a este estudio y se realizaron en la ciudad de Natal en la Universidade Federal do Rio Grande do Norte, en el período de 27 a 31 de enero de 2014. de una idea o concepto clave del texto (75\% nivel 3) y los del $4^{\circ}$ año identificaron más de una idea y lo hicieran con sus propias palabras (75\% nivel 4), por ejemplo:

F3 ( $1^{\circ}$ año): Presenta un estudio innovador (nivel 2, sólo identifican una de las ideas) F6 ( $4^{\circ}$ año): El texto aborda la influencia que la sustancia hipoclorito de sodio 
puede traer en la piel de ratones a partir de un estudio hecho por una institución reconocida, sin presentar datos del uso en seres humanos (nivel 4, expresa con sus propias palabras la información más importante haciendo interacciones entre las ideas principales del texto).

Destacan que el texto presenta de forma más clara el problema principal y que los mensajes implícitos en el título y en las imágenes del texto pueden llevar al uso inadecuado del producto comercial.

\section{Categoría 2: Identificación del propósito del autor}

La mayoría de los futuros profesores del $1^{\circ}$ año (87,5\% nivel 3) considera que el interés del autor es divulgar un estudio científico y promover un beneficio a la sociedad, creen que lo hacen de forma imparcial y neutra, pues se basan en una investigación impartida en una universidad reconocida, como por ejemplo: "F3 ( $1^{\circ}$ año): Con la finalidad de presentar un nuevo estudio que ayudará a las personas (nivel 3, supone que la noticia es para informar de una manera neutra e imparcial)".

Cabe destacar que este grupo (del $1^{\circ}$ año) no había cursado el componente Historia y Filosofía de la Ciencia que es ofrecido como asignatura obligatoria en el curso de magisterio en esta universidad. En este componente del currículo de formación docente se discute, entre otros puntos, el papel de la ciencia en la construcción del conocimiento.

La mayoría de los alumnos del final del curso (75\% nivel 4) identificaron otro interés además de divulgar la noticia, pero no fueron capaces de inferir y expresar concretamente el propósito.

\section{Categoría 3: Identificación de las suposiciones y puntos de vista del autor}

Esta categoría nos parece una de las más significativas pues exige la habilidad cognitiva de inferir a partir de los datos y reconocer que hay una ideología presente en el texto. Así, 62,5\% de los futuros profesores del $1^{\circ}$ año se situaron en el nivel 4 , hicieron algunas suposiciones sobre posibles ideas inferidas del texto: "F9 ( $1^{\circ}$ año): El texto remite la idea de que hay esperanza de un tratamiento para rejuvenecimiento de piel a través de algo sencillo y económico (nivel 4, hace suposición razonable, identifica el punto de vista del autor pero no es justificado)".

Aunque los participantes reconozcan la existencia de otras intenciones del texto revelando la creencia en la ciencia experimental y en el uso de un producto comercial que puede traer beneficios, no la justifican. Por otro lado, el grupo del último año ha elaborado mejores argumentos (37,5\% nivel 5 y 25\% nivel 4) donde identifican puntos de vista antagónicos presentados en el texto y destacan los intereses de grupos sociales con sus ideologías.

F7 ( $4^{\circ}$ año): El autor lo escribe con el objetivo de impactar al lector utilizando un argumento basado en la idoneidad de los puntos de vista científicos y la contradicción con el saber popular (nivel 5, hace una suposición razonable, identifica y justifica el punto de vista del autor a partir del texto).

\section{Categoría 4: Formulación de una pregunta científica o diseño de un experimento para comprobar la información del texto}

En esta categoría obtuvimos los niveles más bajos, tanto en los futuros profesores del $1^{\circ}$ año como en los del último, lo que demuestra la dificultad que tienen para diseñar experimentos. Los del $1^{\circ}$ año (75\% nivel 2) plantearon experimentos generales o incoherentes con el tema del texto. "F4 ( $1^{\circ}$ año): Probar en metales o alimentos y dependiendo del resultado avanzar en la investigación (nivel 1, plantea un experimento irrelevante e incoherente con el tema)". 
Los del último año $(62,5 \%$ nivel 2) hicieron propuestas que consistian en reproducir los resultados del artículo científico o de utilizar el mismo método en humanos. "F1 (4 año): Yo utilizaría un material con propiedades semejantes al de la piel y la bañaría con la misma cantidad de sustancia sugerida en el artículo y observaría sus efectos (nivel 2, plantea un experimento muy general)".

Se observa que los participantes no hicieron preguntas generadoras de nuevos problemas de investigación y que tuvieron dificultad en proponer un experimento para comprobar la información del texto. Además, no consideraron la existencia de un protocolo científico y aspectos éticos a ser considerados antes de realizar experimentos con seres humanos.

\section{Categoría 5: Identificación de datos y pruebas}

En esta categoría se esperaba que los estudiantes fueran capaces de analizar y evaluar la credibilidad de la información presentada movilizando sus conocimientos científicos, posicionándose a favor o en contra las afirmaciones del texto. La totalidad de alumnos del $1^{\circ}$ año no validó la información del texto presentando una posición crédula con la información sin movilizar sus propios conocimientos, situándose en el nivel 2 de esta categoría.

En el caso de los alumnos del último curso los resultados fueron: $50 \%$ en el nivel 2; $25 \%$ en el nivel 3 ya que se cuestionaron si las informaciones tenían validez científica, aunque no presentaron ningún argumento. Finalmente, un 25\% se situó en el nivel 5 haciendo la distinción entre hechos, argumentos científicos $\mathrm{y}$ opiniones del texto. Y por tanto, sacan conclusiones teniendo en cuenta la información y demuestran capacidad para analizarla y evaluarla objetivamente. "F3 ( $1^{\circ}$ año): El texto prueba que los ratones expuestos a radioterapia tuvieron sus células renovadas, mejorando su apariencia (nivel 2 , saca conclusiones a partir de informaciones del texto y no se refiere a las evidencias)".
Algunos de los participantes del $4^{\circ}$ año, valoraron la información basándose en sus propios conocimientos y propusieron leer el artículo científico original para juzgar la credibilidad de la información.

F6 (4 $4^{\circ}$ año): Se observa por los datos, gráficos, fotos y los comentarios de los científicos, que los daños inflamatorios en los ratones no fueron abordados en el texto del periódico (nivel 5, hace distinción entre argumentos científicos y opiniones del texto, sacando las conclusiones por un razonamiento sensato, analizando y evaluando la información objetivamente).

\section{Categoría 6: Argumentación de conclusiones a partir de pruebas}

En esta categoría los alumnos del $1^{\circ}$ año llegan a conclusiones a partir de conocimientos cotidianos sin activar los conocimientos científicos (62,5\% nivel 2). Además, tuvieron dificultad en buscar información en Internet para contrastar la información que ponía en el artículo. Los del $4^{\circ}$ año, por el contrario, mostraron una mayor capacidad para confrontar sus conocimientos de ciencia y la información del texto, a la vez, mostraron mayor agilidad en la búsqueda de información a través de Internet. Estos alumnos participaron activamente en los debates realizados en el aula donde se puso de manifiesto las fragilidades del texto y las posibles implicaciones que la noticia podría provocar.

Basado en el posible uso inadecuado de los productos comerciales, algunos alumnos del $1^{\circ}$ año hicieron cálculos de dilución de la solución del hipoclorito de sodio para demostrar que su uso puede ser un gran problema para aquellos que intentaran hacerlo en casa en función de las concentraciones del hipoclorito en soluciones de productos comerciales. Por otro lado, los participantes del $4^{\circ}$ año no utilizaron cálculos matemáticos para justificar los posibles daños en la piel. Elaboraron modelos microscópicos 
de las interacciones del producto sin necesitar hacer cálculos.

\section{Conclusiones}

Los resultados de esta investigación muestran que los futuros profesores, independientemente del período en el curso, revelan cierta facilidad para identificar las ideas principales del texto. Sin embargo, aquellos que se encuentran en el inicio de sus estudios tuvieron mayores dificultades para reconocer las ideas implícitas relacionadas con la imagen de la ciencia, y por lo tanto, en identificar las suposiciones y puntos de vista del autor. Consideran que el interés del autor es divulgar un estudio científico y promover un beneficio a la sociedad, y creen que lo hacen de forma imparcial y neutra. El hecho de que la investigación la haya realizado una universidad reconocida le confiere credibilidad, y los participantes confían en la información escrita sin necesidad de buscar pruebas en el texto o en otras fuentes (PHILLIPS; NORRIS, 1999). Algunos alumnos del último año, sí fueron capaces de identificar puntos de vista antagónicos en el artículo, y detectar intereses de grupos sociales en el mismo. A pesar de ello, la mayoría de los participantes, tanto de primer como del último año, mostraron una visión ingenua sobre la intencionalidad del autor. Se demuestra así la dificultad de los futuros docentes en hacer análisis críticos y analíticos de textos. Este aspecto se debe tener en cuenta en los programas de formación y fomentar actividades que promuevan el desarrollo del pensamiento crítico en los futuros profesores. De esta manera los docentes estarán capacitados para ayudar a sus alumnos de secundaria a tener estrategias para analizar la credibilidad de diferentes fuentes de información (CLARK; SLOTTA, 2000).

Cabe destacar la dificultad que los futuros profesores de manera independiente del curso mostraron en proponer experimentos o preguntas para validar o refutar las informaciones presentadas en el texto y para argumentar sus conclusiones. Este mismo problema se había detectado en secundaria en estudios anteriores (OLIVERAS; MÁRQUEZ; SANMARTÍ, 2013). Es muy importante que los futuros profesores sepan diseñar experimentos científicos en relación a fenómenos cotidianos. Probablemente hay un problema en su formación en el sentido de estimular su creatividad para diseñar o adecuar experimentos para ser trabajados en el aula evitando que sean rehenes de los manuales.

Otro aspecto a destacar es la dificultad que mostraron los alumnos de primer año en identificar datos y pruebas en el texto. Elaboraron conclusiones basadas en informaciones del texto no relevantes, y no consideraron si eran evidencias o no. Por otro lado, la mitad de los alumnos de último año sí se refirieron a las pruebas que daba el texto, aunque pocos fueron capaces de distinguir explícitamente entre hechos, argumentos científicos y opiniones y sacar conclusiones a partir de la información que disponían. Según Nicolaidou y otros autores (2011), la cooperación entre los estudiantes mejora la identificación y el análisis de las pruebas. No podemos asegurar que los alumnos de último año hayan tenido mejores resultados en este aspecto debido a la mayor participación en los debates de clase, pero quizás, este aspecto puede haber influido. Así pues, consideramos necesario trabajar en las clases y en grupos cooperativos, textos de prensa con contenido científico para ayudar a los alumnos a diferenciar entre hechos, pruebas, argumentos científicos y opiniones, y poder así buscar mejores conexiones entre las afirmaciones y las ideas científicas que van relacionadas (MCNEILL; KRAJCIK, 2008).

Cabe destacar que los participantes no están habituados a este tipo de actividad, la cual requiere de ellos una acción activa en el proceso: cuestionar y comparar sus modelos mentales y diseñar experimentos. Sería necesario realizar más actividades de este tipo en las clases para fomentar la 
lectura crítica y la reflexión sobre problemas sociales relevantes. Por eso, valoramos positivamente la reflexión realizada después de la actividad pues creemos que la discusión y la argumentación de las ideas en pequeños grupos los han ayudado a ser conscientes de sus propias dificultades en analizar críticamente un texto de prensa.

\section{Referencias}

ABRAMI, Philip et al. Strategies for teaching students to think critically: a meta-analysis. Review of Educational Research, Pennsylvania, v. 85, n. 2, p. 275-314, 2015.

AGENCIA Hélice. Água sanitária ajuda a rejuvenescer, aponta estudo. Portal Terra, 16 dez. 2013. Disponible em: <http://beleza. terra.com.br/sua-pele/para-sua-pele/banho-com-agua-sanitaria-poderejuvenescer-aponta-estudo>. Acceso en: 30 dic. 2013.

ARCHILA, Pablo Antonio. La investigación en argumentación y sus implicaciones en la formación inicial de profesores de ciencias. Revista Eureka sobre Enseñanza y Divulgación de las Ciencias, Cádiz, v. 9, n. 3, p. 361-375, 2012.

BARDÍN, Laurence. Análisis de contenido. Madrid: Akal, 1986.

BAILIN, Sharon. Critical thinking and science education. Science \& Education, Netherlands, v. 11, n. 4, p. 361-375, 2002.

BAKTHIN, Mikhail. Estética da criação verbal. São Paulo: Martins Fontes, 2000.

BARTZ, Wayne R. Teaching skepticism via the CRITIC acronym and the skeptical inquirer. Skeptical Inquirer, Amherst, v. 26, n. 5, p. 42-44, 2002.

BORIN, Graziele; BECKER, Alex; OLIVEIRA, Elias. A história do hipoclorito de sódio e a sua importância como substância auxiliar no preparo químico mecânico de canais radiculares. Revista de Endodontia, Pesquisa e Ensino, Santa Maria, v. 3, n. 5, p. 1-5, 2007.

BRAUND, Martin et al. First steps in teaching argumentation: a South African study. International Journal of Educational Development, v. 33, n. 2, p. 175-184, 2013.

CETIN, Pinar et al. The quality of pre-service science teachers' argumentation: influence of content knowledge. Journal Science Teacher Education, v. 25, n. 3, p. 309-331, 2014.

CLARK, Douglas; SLOTTA, James. Evaluating media-enhancement and source authority on the internet: the knowledge integration environment. International Journal of Science Education, London, v. 22, n. 8, p. 859-871, 2000.

CRIPPEN, kent. Argument as professional development: impacting teacher knowledge and beliefs about science. Journal Science Teacher Education, v. 23, n. 8, p. 847-866, 2012.

DIAKIDOY, Irene et al. Forming a belief: the contribution of comprehension to the evaluation and persuasive impact of argumentative text. British Journal of Educational Psychology, v. 85, n. 3, p. 300-315, 2015.

DRIVER, Rosalind et al. Establishing the norms of scientific argumentation in classroom. Science Education, New Jersey, v. 84, n. 3, p. 287-312, 2000

ENNIS, Robert. Critical Thinking. New York: Prentice Hall, 1996.

ERDURAN, Sibel et al. Learning to teach argumentation: case studies of pre-service secondary science teachers. Eurasia Journal of Mathematics, Science and Technology Education, Ankara, v. 2, n. 2, p. 1-14, 2006.

FISHMAN, Barry et al. Linking teacher and student learning to improve professional development in systemic reform. Teaching and Teacher Education, v. 19, n. 6, p. 643-658, 2003.

GARRITZ, Andoni. Argumentación en una nueva asignatura: ciencia y sociedad. Educación Química, México D. F., v. 20, n. 2, p. 98-101, 2009. 
GILBERT, John K.; BULTE, Astrid M. W.; PILOT, Albert. Concept development and transfer in context-based science education. International Journal of Science Education, London, v. 33, n. 6, p. 817-837, 2011.

HAGGER, Paul; KAYE, Michael. Critical thinking in teacher education: a process-oriented research agenda. Australian Journal of Teacher Education, Perth, v. 17, n. 2, p. 26-33, 1992.

HAGGER, Paul et al. Teaching critical thinking in undergraduate science courses. Science \& Education, Netherlands, v. 12, n. 3, p. 303-313, 2003.

HALKIA, Krystallia; MANTZOURIDIS, Dimitris. Students' views and attitudes towards the communication code used in press articles about science. International Journal of Science Education, London, v. 27, n. 12, p. 1395-1411, 2005.

JARMAN, Ruth; MCCLUNE, Billy. Developing students' ability to engage critically with science in the news: identifying elements of the 'media awareness' dimension. Curriculum Journal, London, v. 21, n. 1, p. 47-64, 2010.

JIMÉNEZ-ALEIXANDRE, María Pilar; DÍAZ BUSTAMANTE, Joaquín. Discurso de aula y argumentación en clases: cuestiones teóricas y metodológicas. Enseñanza de las Ciencias, Barcelona, v. 21, n. 3, p. 359-370, 2003.

JIMÉNEZ-ALEIXANDRE, María Pilar; ERDURAN, Sibel. Argumentation in science education: an overview. In: JIMÉNEZ-ALEIXANDRE, María Pilar; ERDURAN, Sibel (Ed.). Argumentation in science education: perspectives from classroom-based research. New York: Springer, 2008. p. 3-28.

JIMÉNEZ-ALEIXANDRE, María Pilar; REIGOSA, Carlos. Contextualizing practices across epistemic levels in the chemistry laboratory. Science Education, New Jersey, v. 90, n. 4, p. 707-733, 2006.

KANG, Hosun; ANDERSON, Charles. Supporting pre-service science teachers' ability to attend and respond to student thinking by design. Science Education, New Jersey, v. 99, n. 5, p. 863-895, 2015.

KELLY, Gregory. Inquiry, active and epistemic practice. In: DUSCHL, Richard; GRANDY, Richard (Ed.). Teaching scientific inquiry: recommendations for research and implementation. Rotterdam: Sense, 2008. p. 99-107.

KENNEDY, Mellen; FISHER, Michelle; ENNIS, Robert. Critical thinking: literature review and needed research. In: IDOL, Lorna; JONES, Beau (Ed.). Educational values and cognitive instruction: implications for reform. New York: Lawrence Erlbaum, 1991. p. 11-40.

KUHN, Deanne. Science as argument: implications for teaching and learning scientific thinking. Science Education, New Jersey, v. 77, n. 3, p. 319-337, 1993.

KUHN, Deanne. Teaching and learning science as argument. Science Education, New Jersey, v. 94, n. 5, p. 810-824, 2010.

LAWSON, A. Basic inferences of scientific reasoning, argumentation and discovery. Science Education, New Jersey, v. 94, n. 2 , p. 336-364, 2010.

LEE, Sung-Tao; LIN, Huann-Shyang. Using argumentation to investigate science teacher's teaching practices: the perspective of instructional decisions and justifications. International Journal of Science and Mathematics Education, Taiwan, v. 3, n. 3, p. 429-461, 2005.

LEUNG, Thomas et al. Topical hypochlorite ameliorates NF-B-mediated skin diseases in mice. The Journal of Clinical Investigation, North Carolina, v. 123, n. 12, p. 5361-5370, 2013.

LINHARES, Salete; PASSOS, Luciana. 0 espaço para a argumentação no ensino superior de Química. Educación Química, México D. F., v. 20, p. 104-110, 2009.

LOUCKS-HORSLEY, Susan et al. Designing professional development for teachers of science and mathematics. California: Corwin, 2003.

MANGIANTE, Elaine. Planning science instruction for critical thinking: two urban elementary teachers' responses to a state science assessment. Education Science, Switzerland, v. 3, n. 3, p. 222-258, 2013.

MÁRQUEZ, Conxita; PRAT, Angels. Leer en clase de ciencias. Enseñanza de las Ciencias, Barcelona, v. 23, n. 3, p. 431-440, 2005. 
MCCLUNE, Billy; Alexander, Joy. Learning to read with a critical eye: cultivating discerning readers of media reports with a science component. Science, Literacy and Learning, London, v. 97, p. 359, 2015.

MCDONALD, Christine. The influence of explicit nature of science and argumentation instruction on preservice primary teachers' views of nature of science. Journal of Research in Science Teaching, Champaing, v. 47, n. 9, p. 1137-1164, 2010.

MCNEILL, Katherine; KRAJCIK, Joseph. Scientific explanations: characterizing and evaluating the effects of teachers' instructional practices on student learning. Journal of Research in Science Teaching, Champaing, v. 45, n. 1, p. 53-78, 2008.

NEWTON, Paul; DRIVER, Rosalind; OSBORNE, Jonathan. The place of argumentation in the pedagogy of school science. International Journal of Science Education, London, v. 21, n. 5, p. 553-576, 1999.

NICOLAIDOU, lolie et al. A framework for scaffolding students' assessment of the credibility of evidence. Journal of Research in Science Teaching, Champaing, v. 48, p. 711-744, 2011.

NORRIS, Stephen; PHILLIPS, Linda; KORPAN, Connie. University students' interpretation of media reports of science and its relationship to background knowledge, interest and reading difficulty. Public Understanding of Science, London, v. 12, p. 123145, 2003.

NOVAK, John; DETLOFF, Janet. Developing critical thinking skills in community college students. Journal of College Science Teaching, Virginia, v. 19, n. 1, p. 22-25, 1989.

NUSSBAUM, Michael. Scaffolding argumentation in the social studies classroom. The Social Studies, Philadelphia, v. 93, n. 2, p. 79-83, 2002.

OGBORN, Jon et al. Formas de explicar: la enseñanza de las ciencias en secundaria. Madrid: Aula XXI, 1998.

OLIVERAS, Begoña. La lectura crítica a la clase de ciencias: propostes, dificultats i perfils lectores. 2014. 155 p. Tesis (Doctorado) - Departament de Didàctica de la Matemática i de les Ciències Experimentals, Universidad Autónoma de Barcelona, Bellaterra. 2014.

OLIVERAS, Begoña; MÁRQUEZ, Conxita; SANMARTÍ, Neus. Students' attitudes to information in the press: critical reading of a newspaper article with scientific content. Research in Science Education, New York, v. 44, n. 4, p. 603-626, 2014.

OLIVERAS, Begoña; MÁRQUEZ, Conxita; SANMARTÍ, Neus. The use of newspaper articles as a tool to develop critical thinking in science classes. International Journal of Science Education, London, v. 35, n. 6, p. 885-905, 2013.

OLIVERAS, Begoña; SANMARTÍ, Neus. La lectura como medio para desarrollar el pensamiento crítico. Educación Química, México D. F., v. 20, n. 1, p. 233-245, 2009.

OSBORNE, Jonathan. Scientific practices and inquiry in the science classroom. In: LEDERMAN, Norman; ABELL, Sandra (Ed.). Handbook of research on science education. New York: Routledge, 2014. p. 579-599.

PAUL, Richard; ELDER, Linda. A guide for educators to critical thinking competency standards. California: Foundation for Critical Thinking, 2005.

PHILLIPS, Linda; NORRIS, Stephen. Interpreting popular reports of science: what happens when the reader's world meets the world on paper? International Journal of Science Education, London, v. 21, n. 3, p. 317-327, 1999.

PLUMMER, Julia; OZCELIK, Arzu. Preservice teachers developing coherent inquiry investigations in elementary astronomy. Science Education, New Jersey, v. 99, n. 5, p. 932-957, 2015.

RUIZ ORTEGA, Francisco; TAMAYO, Oscar; MÁRQUEZ, Conxita. La argumentación en clase de ciencias, un modelo para su enseñanza. Educação e Pesquisa, São Paulo, v. 41, n. 3, p. 629-645, 2015.

SAMPSON, Victor; BLANCHARD, Margaret. Science teachers and scientific argumentation: trends in views and practice. Journal of Research in Science Teaching, Champaing, v. 49, n. 9, p.1122-1148, 2012.

SAMPSON, Victor; GERBINO, Francesca. Two instructional models that teachers can use to promote \& support scientific argumentation in the biology classroom. The American Biology Teacher, California, v. 72, n. 7, p. 427-431, 2010. 
SARDÁ, Anna; SANMARTÍ, Neus. Enseñar a argumentar científicamente: un reto de las clases de ciencias. Enseñanza de las Ciencias, Barcelona, v. 18, n. 3, p. 405-422, 2000.

SCHWARZ, Christina. Developing preservice elementary teachers' knowledge and practices through modeling-centered scientific inquiry. Science Education, New Jersey, v. 93, n. 4, p. 720-744, 2009.

SIMON, Shirley et al. Learning to teach argumentation: research and development in the science classroom. International Journal of Science Education, London, v. 28, n. 2/3, p. 235-260, 2006.

SOLÉ, Isabel. Estratégias de leitura. Porto Alegre: Artmed, 1998.

STIPCICH, María et al. El lugar de la argumentación en la formación de profesores de Ciencias, Revista Chilena de Educación Científica, Santiago de Chile, v. 6, n. 1, p. 67-74, 2006.

TAVARES, Marina et al. Articulation of conceptual knowledge and argumentation practices by high school students in evolution problems. Science \& Education, Netherlands, v. 19, n. 6-8, p. 573-598, 2010.

VYGOTSKY, Lev. Pensamiento y lenguaje. Barcelona: Paidós. 1977.

WOODS, David. Transana. Madison: Wiscosin Center for Education Research, 2002.

ZEMBAL-SAUL, Carla et al. Scaffolding preservice science teachers' evidence-based arguments during an investigation of natural selection. Research in Science Education, Netherlands, v. 32, n. 4, p. 437-463, 2002.

ZOHAR, Anat. Science teacher education and professional development in argumentation. In: ERDURAN, Sibel; JIMÉNEZALEIXANDRE, Maria Pilar (Ed.) Argumentation in science education: perspectives from classroom-based research. New York: Springer, 2007. p. 245-268.

ZOHAR, Anat; NEMET, Flora. Fostering students' knowledge and argumentation skills through dilemmas in human genetics. Journal of Research in Science Teaching, Champaing, v. 39, n. 1, p. 35-62, 2002.

ZOLLER, Uri et al. The disposition towards critical thinking of high school and university science students: an inter-intra israeliitalian study. International Journal of Science Education, London, v. 22, n. 6, p. 571-582, 2000.

Recibido en: 28.03.2016

Aprobado en: 21.06.2016

Márcia Gorette Lima da Silva es doctora en Educación, profesora del Instituto de Química y coordinadora del Doctorado en Enseñanza de las Ciencias y Matemática de la Universidad Federal del Rio Grande do Norte. Miembro del Grupo de Investigación Química, Enseñanza y Aprendizaje.

Conxita Marquez Bargalló es doctora en Didáctica de las Ciencias Experimentales por la Universidad Autónoma de Barcelona y profesora del Departamento de Didáctica de la Matemática y de las Ciencias Experimentales de la misma universidad. Miembro del Grupo de Investigación Lenguaje y Enseñanza de las Ciencias.

Begonya Oliveras Prat es doctora en Didáctica de las Ciencias Experimentales por la Universidad Autónoma de Barcelona, profesora del Departamento de Didáctica de la Matemática y de las Ciencias Experimentales de la misma universidad y profesora de secundaria del Institut Obert de Catalunya. Miembro del Grupo de Investigación Lenguaje y Enseñanza de las Ciencias. 